BNL-114551-2017-IR

CBETA/017

June 2017

\title{
Transverse forces in the CBETA v6/v6.5 magnets
}

\author{
S. Brooks
}

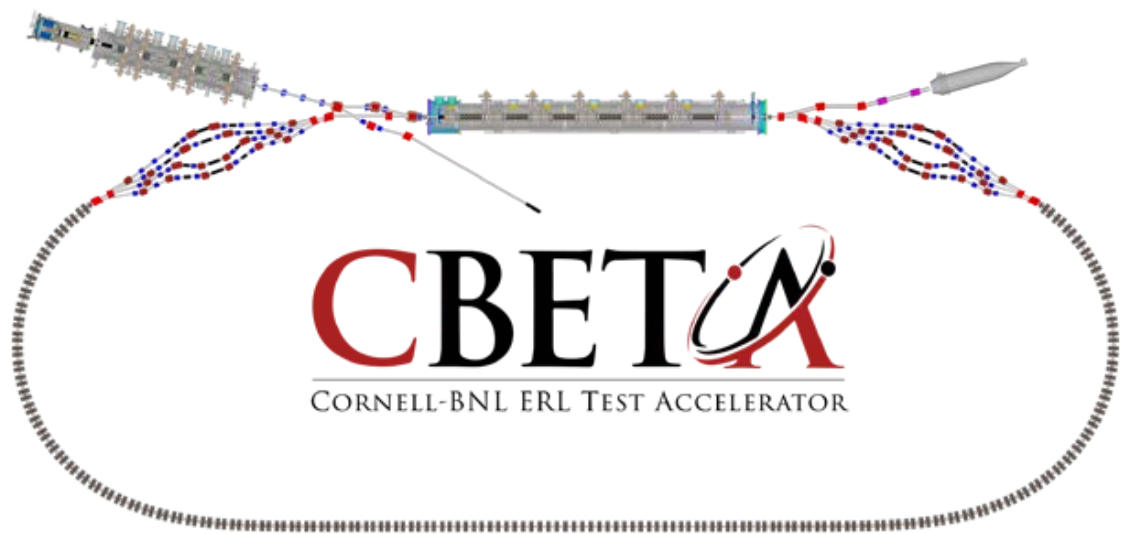

Collider-Accelerator Department, Brookhaven National Laboratory, Upton NY 11973

U.S. Department of Energy

Office of Science, Office of Nuclear Physics

Cornell Laboratory for Accelerator-Based Sciences and Education, Ithaca, NY 14850

Funded by NYSERDA contract 102192

Notice: This document has been authorized by employees of Brookhaven Science Associates, LLC under Contract No. DE-SC0012704 with the U.S. Department of Energy. The United States Government retains a non-exclusive, paid-up, irrevocable, world-wide license to publishor reproduce the published form of this document, or allow others to do so, for United States Government purposes. 


\section{DISCLAIMER}

This report was prepared as an account of work sponsored by an agency of the United States Government. Neither the United States Government nor any agency thereof, nor any of their employees, nor any of their contractors, subcontractors, or their employees, makes any warranty, express or implied, or assumes any legal liability or responsibility for the accuracy, completeness, or any third party's use or the results of such use of any information, apparatus, product, or process disclosed, or represents that its use would not infringe privately owned rights. Reference herein to any specific commercial product, process, or service by trade name, trademark, manufacturer, or otherwise, does not necessarily constitute or imply its endorsement, recommendation, or favoring by the United States Government or any agency thereof or its contractors or subcontractors. The views and opinions of authors expressed herein do not necessarily state or reflect those of the United States Government or any agency thereof. 


\section{Transverse Forces in the CBETA v6/v6.5 Magnets}

Stephen Brooks 2017-Jun-16 CBETA machine note \#17

\section{Introduction}

These 2D transverse force calculations were done for the following Halbach magnet types in the CBETA design as it currently stands (an implementation of the April 10, 2017 lattice). These magnets are made out of two longitudinally-stacked layers of permanent magnet blocks.

\begin{tabular}{|l|l|l|l|}
\hline Magnet Type & Design Version & Magnet length $(\mathrm{m})$ & Block length $(\mathrm{m})$ \\
\hline QF & v6.5 & 0.133 & 0.0665 \\
\hline BD & v6 & 0.122 & 0.061 \\
\hline BDT1 & v6 & 0.122 & 0.061 \\
\hline BDT2 & v6 & 0.122 & 0.061 \\
\hline QD & v6 & 0.122 & 0.061 \\
\hline
\end{tabular}

There is also a QFH ("QF Half") magnet, which is only a single layer of the QF permanent magnet blocks and a BDH magnet which is similarly a single layer of BD. In the 2D approximation, the forces per block in these magnets should be the same as the respective $Q F$ and $B D$ magnets but because there is only one layer, total forces e.g. on the aluminium surround will be halved.

\section{QF v6.5 Magnet}

The picture below shows the transverse forces on each block as vectors (dark red), when the QF magnet is assembled. The light blue arrows are the magnetisation direction. 


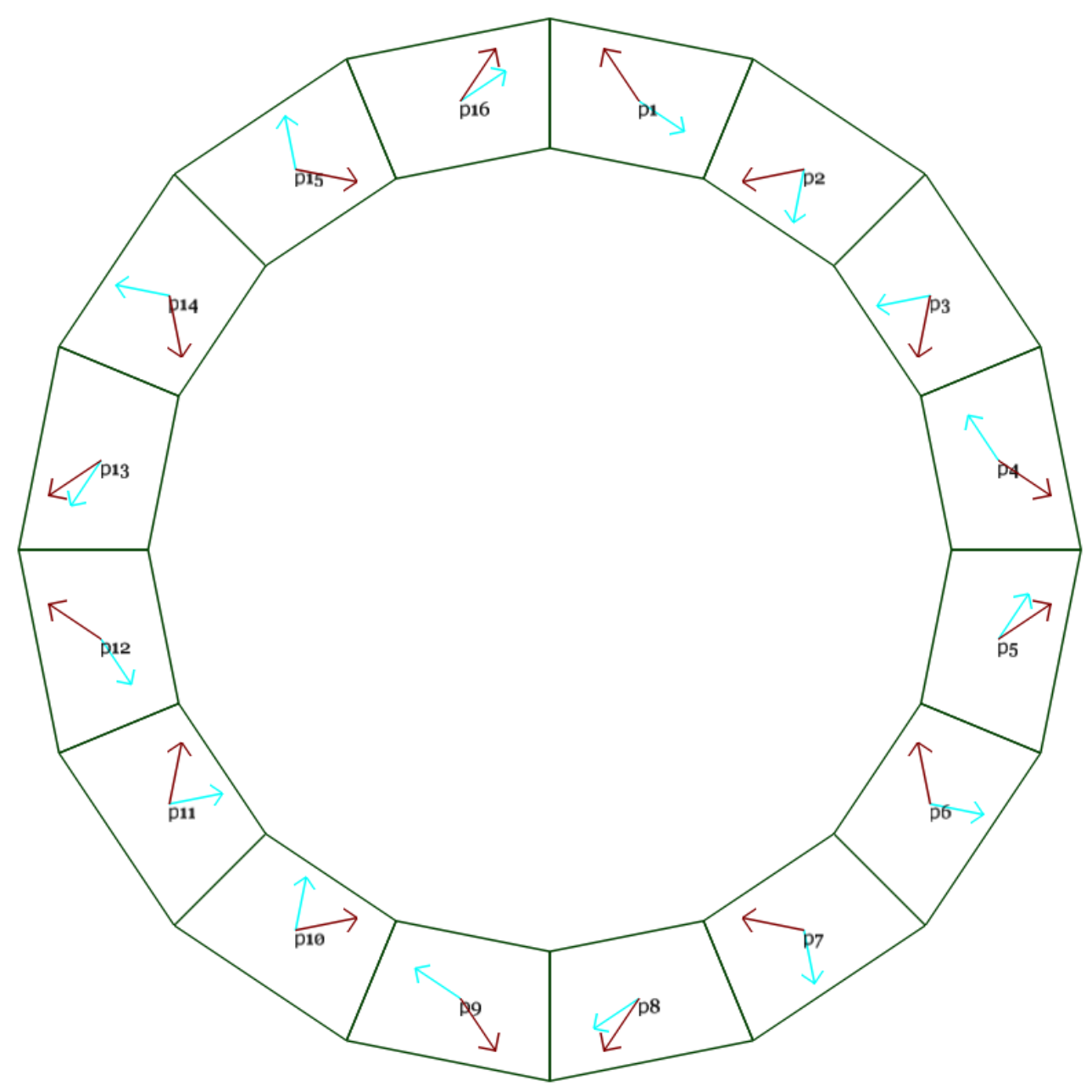

The pieces are labelled arbitrarily p1-p16 so that the corresponding forces can be written in the table below. For actual block type names from the factory, consult the assembly instructions.

The force can be stated as a force per unit length (Newtons/metre), or a force per block, or for the two blocks constituting the total length of the magnet. The latter gives the force on the outer aluminium shell. (Fx,Fy) force vectors are given in each cell.

\begin{tabular}{|l|l|l|l|l|l|}
\hline $\begin{array}{l}\text { Block label in } \\
\text { diagram }\end{array}$ & $\begin{array}{l}\text { Force per unit } \\
\text { length }(\mathrm{N} / \mathrm{m})\end{array}$ & $\begin{array}{l}\text { Force per } \\
\text { block (N) }\end{array}$ & $\begin{array}{l}\text { Force per } \\
\text { block (lbf) }\end{array}$ & $\begin{array}{l}\text { Force full } \\
\text { length (N) }\end{array}$ & $\begin{array}{l}\text { Force full } \\
\text { length (lbf) }\end{array}$ \\
\hline p1 & $(-4090,6103)$ & $(-272,406)$ & $(-61.1,91.2)$ & $(-544,812)$ & $(-122.3,182.5)$ \\
\hline p2 & $(-7223,-1447)$ & $(-480,-96)$ & $(-108,-21.6)$ & $(-961,-192)$ & $(-216,-43.3)$ \\
\hline p3 & $(-1447,-7223)$ & $(-96,-480)$ & $(-21.6,-108)$ & $(-192,-961)$ & $(-43.3,-216)$ \\
\hline p4 & $(6103,-4090)$ & $(406,-272)$ & $(91.2,-61.1)$ & $(812,-544)$ & $(182.5,-122.3)$ \\
\hline p5 & $(6103,4090)$ & $(406,272)$ & $(91.2,61.1)$ & $(812,544)$ & $(182.5,122.3)$ \\
\hline p6 & $(-1447,7223)$ & $(-96,480)$ & $(-21.6,108)$ & $(-192,961)$ & $(-43.3,216)$ \\
\hline p8 & $(-7223,1447)$ & $(-480,96)$ & $(-108,21.6)$ & $(-961,192)$ & $(-216,43.3)$ \\
\hline p9 & $(-4090,-6103)$ & $(-272,-406)$ & $(-61.1,-91.2)$ & $(-544,-812)$ & $(-122.3,-182.5)$ \\
\hline p10 & $(4090,-6103)$ & $(272,-406)$ & $(61.1,-91.2)$ & $(544,-812)$ & $(122.3,-182.5)$ \\
\hline p11 & $(7223,1447)$ & $(480,96)$ & $(108,21.6)$ & $(961,192)$ & $(216,43.3)$ \\
\hline
\end{tabular}




\begin{tabular}{|l|l|l|l|l|l|}
\hline p12 & $(-6103,4090)$ & $(-406,272)$ & $(-91.2,61.1)$ & $(-812,544)$ & $(-182.5,122.3)$ \\
\hline p13 & $(-6103,-4090)$ & $(-406,-272)$ & $(-91.2,-61.1)$ & $(-812,-544)$ & $(-182.5,-122.3)$ \\
\hline p14 & $(1447,-7223)$ & $(96,-480)$ & $(21.6,-108)$ & $(192,-961)$ & $(43.3,-216)$ \\
\hline p15 & $(7223,-1447)$ & $(480,-96)$ & $(108,-21.6)$ & $(961,-192)$ & $(216,-43.3)$ \\
\hline p16 & $(4090,6103)$ & $(272,406)$ & $(61.1,91.2)$ & $(544,812)$ & $(122.3,182.5)$ \\
\hline
\end{tabular}

The magnets will be assembled from two halves, with a vertical split forming a left half and a right half. The table below shows the total force vector on each half when they are in contact.

\begin{tabular}{|l|l|l|l|l|l|}
\hline Subassembly & $\begin{array}{l}\text { Force per unit } \\
\text { length }(\mathrm{N} / \mathrm{m})\end{array}$ & $\begin{array}{l}\text { Force per } \\
\text { layer (N) }\end{array}$ & $\begin{array}{l}\text { Force per } \\
\text { layer (lbf) }\end{array}$ & $\begin{array}{l}\text { Force full } \\
\text { length (N) }\end{array}$ & $\begin{array}{l}\text { Force full } \\
\text { length (lbf) }\end{array}$ \\
\hline Left half & $(13311,0)$ & $(885,0)$ & $(199,0)$ & $(1770,0)$ & $(398,0)$ \\
\hline Right half & $(-13311,0)$ & $(-885,0)$ & $(-199,0)$ & $(-1770,0)$ & $(-398,0)$ \\
\hline
\end{tabular}

\section{BD v6 Magnet}

The picture below shows the transvers forces on each block as vectors (dark red), when the BD magnet is assembled. The light blue arrows are the magnetisation direction.

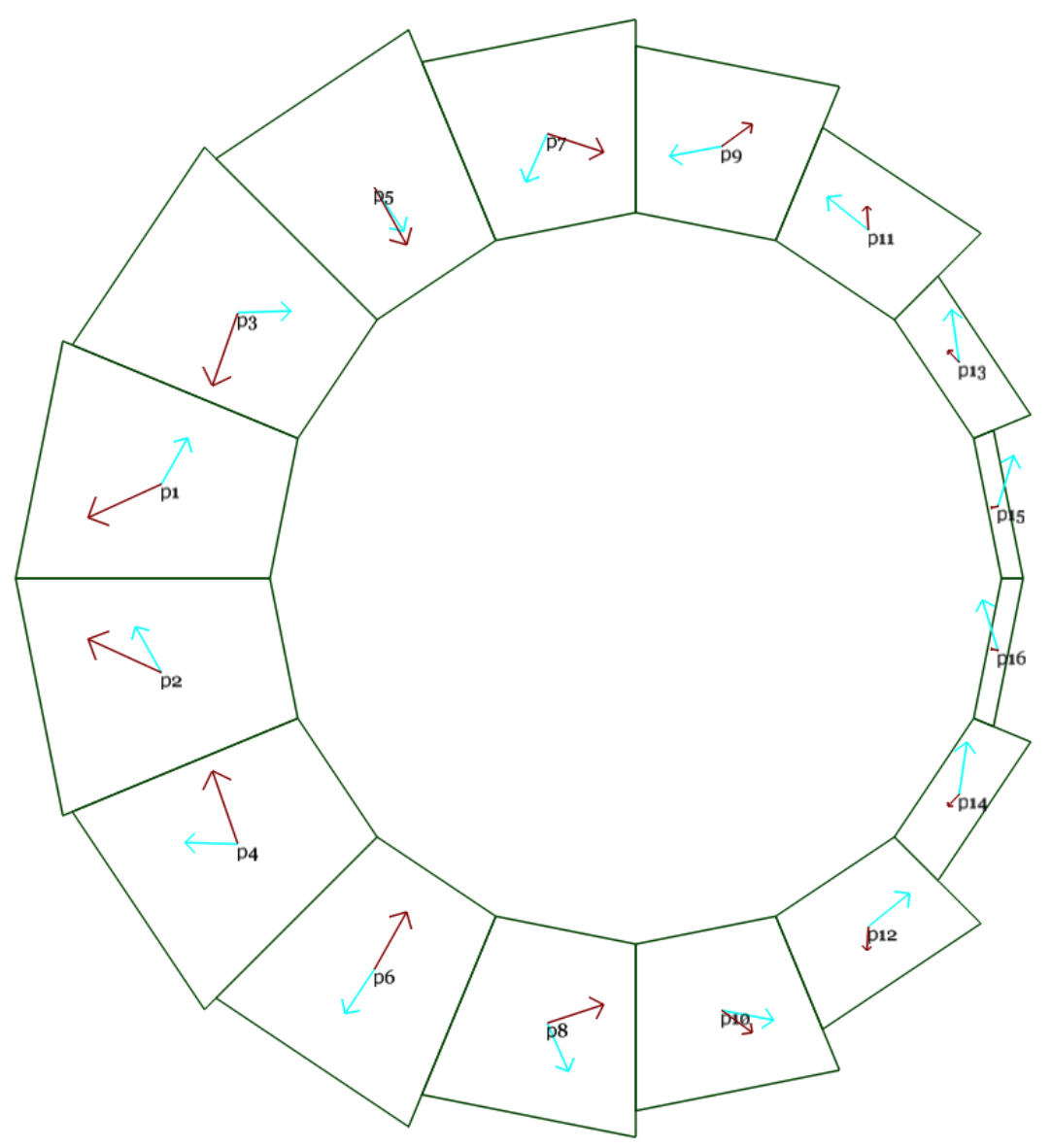


The force vectors on each piece of the BD magnet are written in the table below.

\begin{tabular}{|l|l|l|l|l|l|}
\hline $\begin{array}{l}\text { Block label in } \\
\text { diagram }\end{array}$ & $\begin{array}{l}\text { Force per unit } \\
\text { length }(\mathrm{N} / \mathrm{m})\end{array}$ & $\begin{array}{l}\text { Force per } \\
\text { block (N) }\end{array}$ & $\begin{array}{l}\text { Force per } \\
\text { block (lbf) }\end{array}$ & $\begin{array}{l}\text { Force full } \\
\text { length (N) }\end{array}$ & $\begin{array}{l}\text { Force full } \\
\text { length (lbf) }\end{array}$ \\
\hline p1 & $(-8842,-4046)$ & $(-539,-247)$ & $(-121.2,-55.5)$ & $(-1079,-494)$ & $(-242.5,-111)$ \\
\hline p2 & $(-8842,4046)$ & $(-539,247)$ & $(-121.2,55.5)$ & $(-1079,494)$ & $(-242.5,111)$ \\
\hline p3 & $(-3086,-8837)$ & $(-188,-539)$ & $(-42.3,-121.2)$ & $(-377,-1078)$ & $(-84.6,-242.4)$ \\
\hline p4 & $(-3086,8837)$ & $(-188,539)$ & $(-42.3,121.2)$ & $(-377,1078)$ & $(-84.6,242.4)$ \\
\hline p5 & $(3882,-6923)$ & $(237,-422)$ & $(53.2,-94.9)$ & $(474,-845)$ & $(106.5,-189.9)$ \\
\hline p6 & $(3882,6923)$ & $(237,422)$ & $(53.2,94.9)$ & $(474,845)$ & $(106.5,189.9)$ \\
\hline p7 & $(6782,-2224)$ & $(414,-136)$ & $(93,-30.5)$ & $(827,-271)$ & $(186,-61)$ \\
\hline p8 & $(6782,2224)$ & $(414,136)$ & $(93,30.5)$ & $(827,271)$ & $(186,61)$ \\
\hline p9 & $(3676,2670)$ & $(224,163)$ & $(50.4,36.6)$ & $(449,326)$ & $(100.8,73.2)$ \\
\hline p10 & $(3676,-2670)$ & $(224,-163)$ & $(50.4,-36.6)$ & $(449,-326)$ & $(100.8,-73.2)$ \\
\hline p11 & $(-172,2917)$ & $(-10,178)$ & $(-2.4,40)$ & $(-21,356)$ & $(-4.7,80)$ \\
\hline p12 & $(-172,-2917)$ & $(-10,-178)$ & $(-2.4,-40)$ & $(-21,-356)$ & $(-4.7,-80)$ \\
\hline p13 & $(-1348,1411)$ & $(-82,86)$ & $(-18.5,19.4)$ & $(-164,172)$ & $(-37,38.7)$ \\
\hline p14 & $(-1348,-1411)$ & $(-82,-86)$ & $(-18.5,-19.4)$ & $(-164,-172)$ & $(-37,-38.7)$ \\
\hline p15 & $(-876,-194)$ & $(-53,-12)$ & $(-12,-2.7)$ & $(-107,-24)$ & $(-24,-5.3)$ \\
\hline p16 & $(-876,194)$ & $(-53,12)$ & $(-12,2.7)$ & $(-107,24)$ & $(-24,5.3)$ \\
\hline
\end{tabular}

The table below shows the total force vector on each half of the BD magnet when they are in contact. NB: the forces do not sum to exactly zero due to numerical effects in the simulation.

\begin{tabular}{|l|l|l|l|l|l|}
\hline Subassembly & $\begin{array}{l}\text { Force per unit } \\
\text { length }(\mathrm{N} / \mathrm{m})\end{array}$ & $\begin{array}{l}\text { Force per } \\
\text { layer (N) }\end{array}$ & $\begin{array}{l}\text { Force per } \\
\text { layer (lbf) }\end{array}$ & $\begin{array}{l}\text { Force full } \\
\text { length (N) }\end{array}$ & $\begin{array}{l}\text { Force full } \\
\text { length (lbf) }\end{array}$ \\
\hline Left half & $(-2527,0)$ & $(-154,0)$ & $(-34.7,0)$ & $(-308,0)$ & $(-69.3,0)$ \\
\hline Right half & $(2561,0)$ & $(156,0)$ & $(35.1,0)$ & $(312,0)$ & $(70.2,0)$ \\
\hline
\end{tabular}

\section{BDT1 v6 Magnet}

The picture below shows the transvers forces on each block as vectors (dark red), when the BDT1 magnet is assembled. The light blue arrows are the magnetisation direction. 


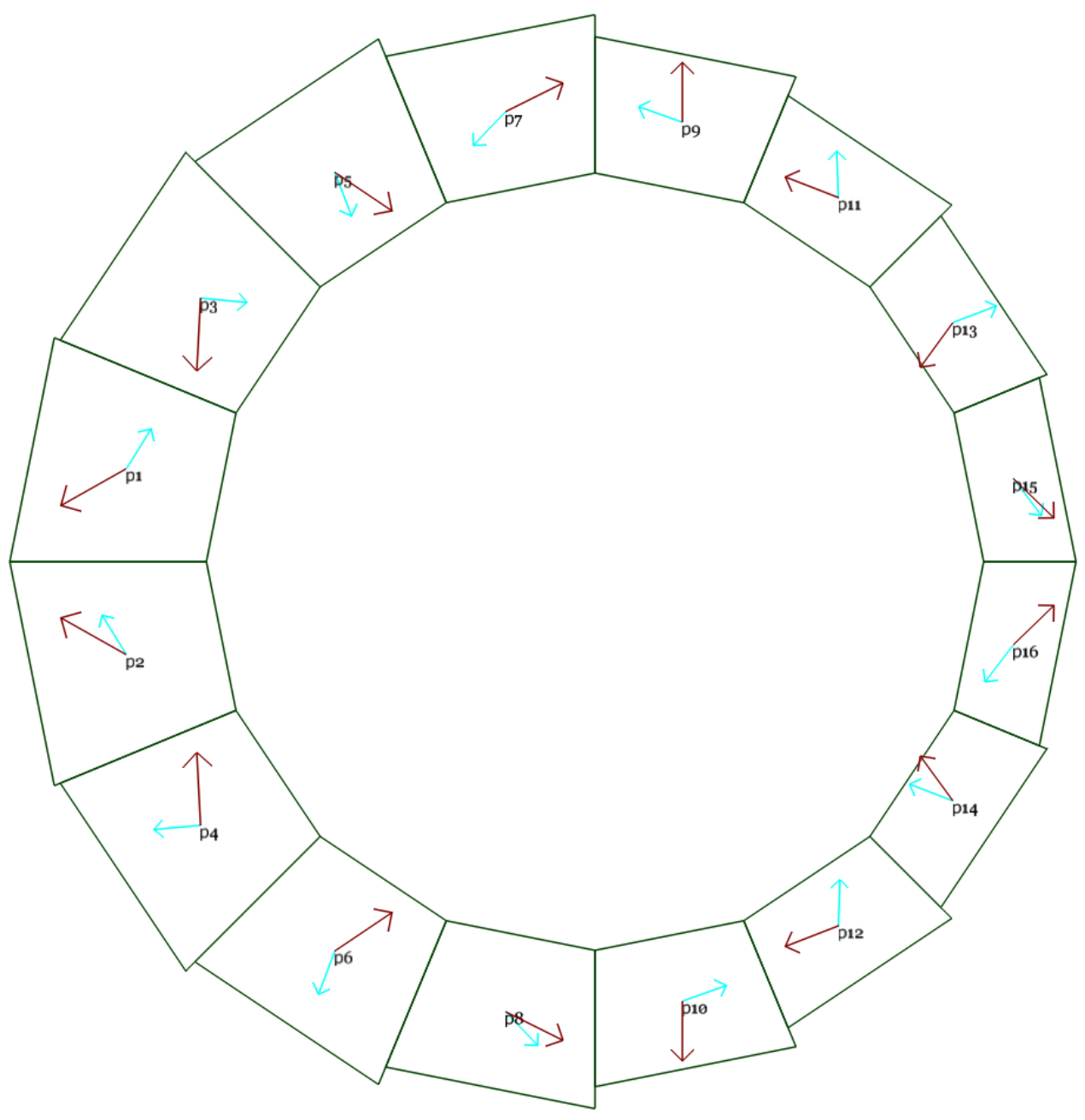

The force vectors on each piece of the BDT1 magnet are written in the table below.

\begin{tabular}{|l|l|l|l|l|l|}
\hline $\begin{array}{l}\text { Block label in } \\
\text { diagram }\end{array}$ & $\begin{array}{l}\text { Force per unit } \\
\text { length }(\mathrm{N} / \mathrm{m})\end{array}$ & $\begin{array}{l}\text { Force per } \\
\text { block (N) }\end{array}$ & $\begin{array}{l}\text { Force per } \\
\text { block (lbf) }\end{array}$ & $\begin{array}{l}\text { Force full } \\
\text { length (N) }\end{array}$ & $\begin{array}{l}\text { Force full } \\
\text { length (lbf) }\end{array}$ \\
\hline p1 & $(-8989,-5069)$ & $(-548,-309)$ & $(-123.3,-69.5)$ & $(-1097,-618)$ & $(-246.5,-139)$ \\
\hline p2 & $(-8989,5069)$ & $(-548,309)$ & $(-123.3,69.5)$ & $(-1097,618)$ & $(-246.5,139)$ \\
\hline p3 & $(-513,-9986)$ & $(-31,-609)$ & $(-7,-136.9)$ & $(-63,-1218)$ & $(-14.1,-273.9)$ \\
\hline p4 & $(-513,9986)$ & $(-31,609)$ & $(-7,136.9)$ & $(-63,1218)$ & $(-14.1,273.9)$ \\
\hline p5 & $(7798,-5243)$ & $(476,-320)$ & $(106.9,-71.9)$ & $(951,-640)$ & $(213.9,-143.8)$ \\
\hline p6 & $(7798,5243)$ & $(476,320)$ & $(106.9,71.9)$ & $(951,640)$ & $(213.9,143.8)$ \\
\hline p7 & $(7876,3922)$ & $(480,239)$ & $(108,53.8)$ & $(961,479)$ & $(216,107.6)$ \\
\hline p8 & $(7876,-3922)$ & $(480,-239)$ & $(108,-53.8)$ & $(961,-479)$ & $(216,-107.6)$ \\
\hline p9 & $(-11,8205)$ & $(-1,501)$ & $(-0.1,112.5)$ & $(-1,1001)$ & $(-0.3,225)$ \\
\hline p10 & $(-11,-8205)$ & $(-1,-501)$ & $(-0.1,-112.5)$ & $(-1,-1001)$ & $(-0.3,-225)$ \\
\hline p11 & $(-7254,2819)$ & $(-442,172)$ & $(-99.5,38.7)$ & $(-885,344)$ & $(-199,77.3)$ \\
\hline p12 & $(-7254,-2819)$ & $(-442,-172)$ & $(-99.5,-38.7)$ & $(-885,-344)$ & $(-199,-77.3)$ \\
\hline p13 & $(-4462,-6118)$ & $(-272,-373)$ & $(-61.2,-83.9)$ & $(-544,-746)$ & $(-122.4,-167.8)$ \\
\hline p14 & $(-4462,6118)$ & $(-272,373)$ & $(-61.2,83.9)$ & $(-544,746)$ & $(-122.4,167.8)$ \\
\hline p15 & $(5553,-5389)$ & $(339,-329)$ & $(76.1,-73.9)$ & $(677,-658)$ & $(152.3,-147.8)$ \\
\hline p16 & $(5553,5389)$ & $(339,329)$ & $(76.1,73.9)$ & $(677,658)$ & $(152.3,147.8)$ \\
\hline
\end{tabular}


The table below shows the total force vector on each half of the BDT1 magnet when they are in contact. NB: the forces do not sum to exactly zero due to numerical effects in the simulation.

\begin{tabular}{|l|l|l|l|l|l|}
\hline Subassembly & $\begin{array}{l}\text { Force per unit } \\
\text { length }(\mathrm{N} / \mathrm{m})\end{array}$ & $\begin{array}{l}\text { Force per } \\
\text { layer (N) }\end{array}$ & $\begin{array}{l}\text { Force per } \\
\text { layer (lbf) }\end{array}$ & $\begin{array}{l}\text { Force full } \\
\text { length (N) }\end{array}$ & $\begin{array}{l}\text { Force full } \\
\text { length (lbf) }\end{array}$ \\
\hline Left half & $(12344,0)$ & $(753,0)$ & $(169.3,0)$ & $(1506,0)$ & $(338.6,0)$ \\
\hline Right half & $(-12349,0)$ & $(-753,0)$ & $(-169.3,0)$ & $(-1507,0)$ & $(-338.7,0)$ \\
\hline
\end{tabular}

\section{BDT2 v6 Magnet}

The picture below shows the transvers forces on each block as vectors (dark red), when the BDT2 magnet is assembled. The light blue arrows are the magnetisation direction.

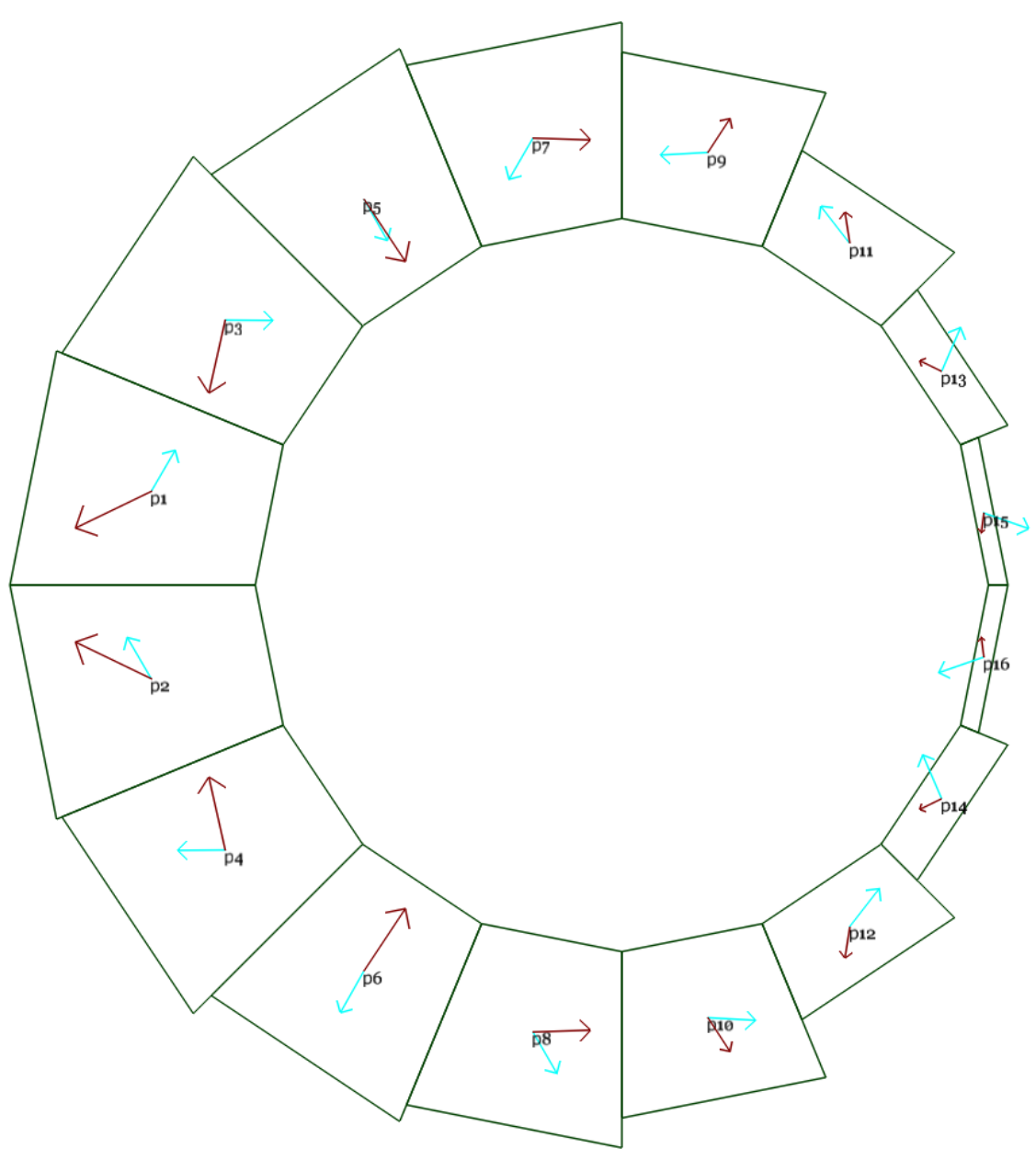

The force vectors on each piece of the BDT2 magnet are written in the table below.

\section{Block label}

in diagram

\section{Force per unit} length $(\mathrm{N} / \mathrm{m})$

\section{Force per} block (N)

\section{Force per} block (lbf)

\section{Force full}

length (N)
Force full length (lbf) 


\begin{tabular}{|l|l|l|l|l|l|}
\hline p1 & $(-10118,-4888)$ & $(-617,-298)$ & $(-138.7,-67)$ & $(-1234,-596)$ & $(-277.5,-134.1)$ \\
\hline p2 & $(-10118,4888)$ & $(-617,298)$ & $(-138.7,67)$ & $(-1234,596)$ & $(-277.5,134.1)$ \\
\hline p3 & $(-2197,-9771)$ & $(-134,-596)$ & $(-30.1,-134)$ & $(-268,-1192)$ & $(-60.3,-268)$ \\
\hline p4 & $(-2197,9771)$ & $(-134,596)$ & $(-30.1,134)$ & $(-268,1192)$ & $(-60.3,268)$ \\
\hline p5 & $(5551,-8360)$ & $(339,-510)$ & $(76.1,-114.6)$ & $(677,-1020)$ & $(152.2,-229.3)$ \\
\hline p6 & $(5551,8360)$ & $(339,510)$ & $(76.1,114.6)$ & $(677,1020)$ & $(152.2,229.3)$ \\
\hline p7 & $(7709,-246)$ & $(470,-15)$ & $(105.7,-3.4)$ & $(940,-30)$ & $(211.4,-6.7)$ \\
\hline p8 & $(7709,246)$ & $(470,15)$ & $(105.7,3.4)$ & $(940,30)$ & $(211.4,6.7)$ \\
\hline p9 & $(2943,4511)$ & $(180,275)$ & $(40.4,61.9)$ & $(359,550)$ & $(80.7,123.7)$ \\
\hline p10 & $(2943,-4511)$ & $(180,-275)$ & $(40.4,-61.9)$ & $(359,-550)$ & $(80.7,-123.7)$ \\
\hline p11 & $(-669,4258)$ & $(-41,260)$ & $(-9.2,58.4)$ & $(-82,519)$ & $(-18.3,116.8)$ \\
\hline p12 & $(-669,-4258)$ & $(-41,-260)$ & $(-9.2,-58.4)$ & $(-82,-519)$ & $(-18.3,-116.8)$ \\
\hline p13 & $(-2945,1443)$ & $(-180,88)$ & $(-40.4,19.8)$ & $(-359,176)$ & $(-80.8,39.6)$ \\
\hline p14 & $(-2945,-1443)$ & $(-180,-88)$ & $(-40.4,-19.8)$ & $(-359,-176)$ & $(-80.8,-39.6)$ \\
\hline p15 & $(-335,-2617)$ & $(-20,-160)$ & $(-4.6,-35.9)$ & $(-41,-319)$ & $(-9.2,-71.8)$ \\
\hline p16 & $(-335,2617)$ & $(-20,160)$ & $(-4.6,35.9)$ & $(-41,319)$ & $(-9.2,71.8)$ \\
\hline
\end{tabular}

The table below shows the total force vector on each half of the BDT2 magnet when they are in contact. NB: the forces do not sum to exactly zero due to numerical effects in the simulation.

\begin{tabular}{|l|l|l|l|l|l|}
\hline Subassembly & $\begin{array}{l}\text { Force per unit } \\
\text { length (N/m) }\end{array}$ & $\begin{array}{l}\text { Force per } \\
\text { layer (N) }\end{array}$ & $\begin{array}{l}\text { Force per } \\
\text { layer (lbf) }\end{array}$ & $\begin{array}{l}\text { Force full } \\
\text { length (N) }\end{array}$ & $\begin{array}{l}\text { Force full } \\
\text { length (lbf) }\end{array}$ \\
\hline Left half & $(1889,0)$ & $(115,0)$ & $(25.9,0)$ & $(231,0)$ & $(51.8,0)$ \\
\hline Right half & $(-2012,0)$ & $(-123,0)$ & $(-27.6,0)$ & $(-245,0)$ & $(-55.2,0)$ \\
\hline
\end{tabular}

\section{QD v6 Magnet}

The picture below shows the transvers forces on each block as vectors (dark red), when the QD magnet is assembled. The light blue arrows are the magnetisation direction. 


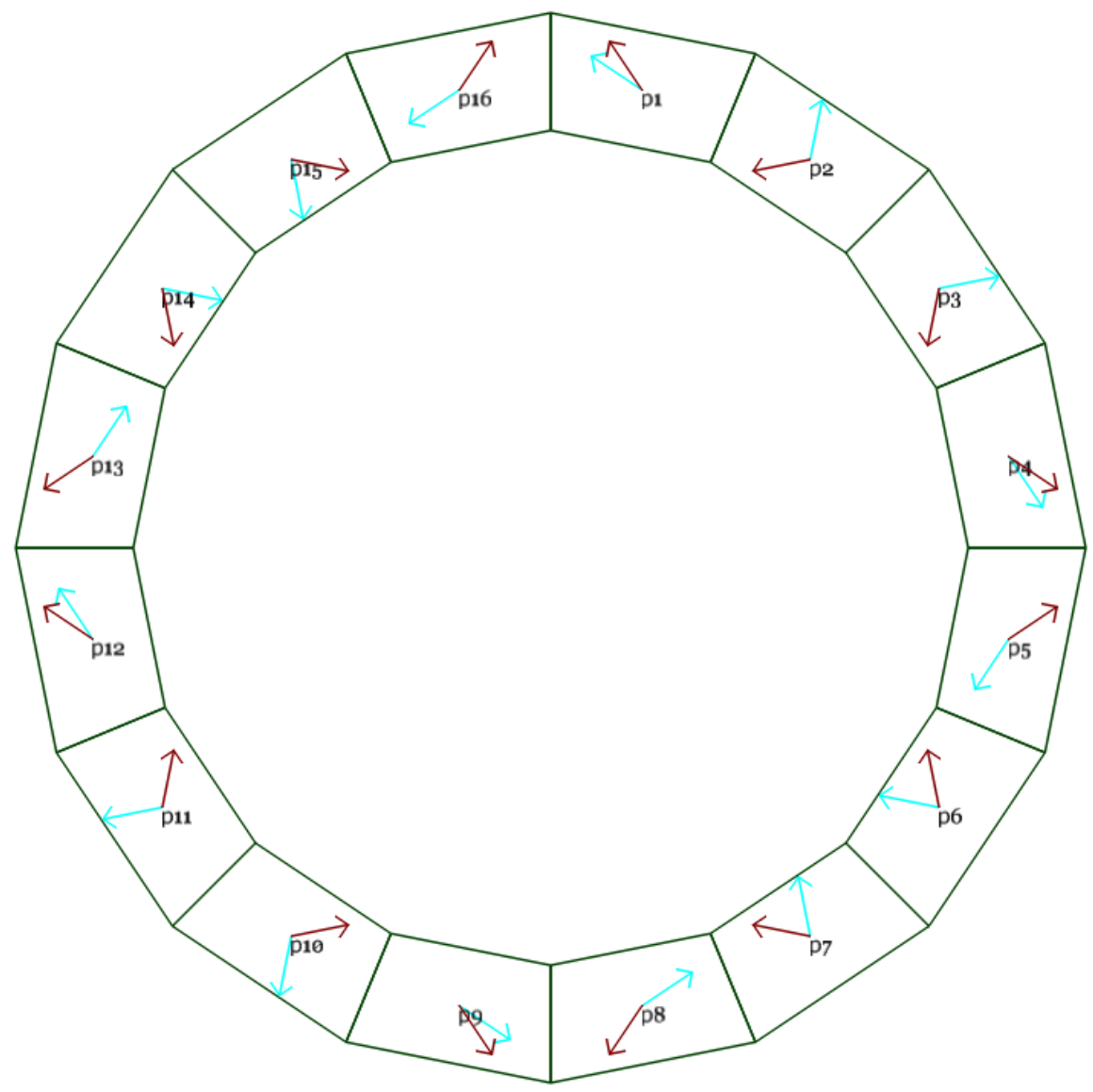

The force vectors on each piece of the QD magnet are written in the table below.

\begin{tabular}{|l|l|l|l|l|l|}
\hline $\begin{array}{l}\text { Block label in } \\
\text { diagram }\end{array}$ & $\begin{array}{l}\text { Force per unit } \\
\text { length }(\mathbf{N} / \mathrm{m})\end{array}$ & $\begin{array}{l}\text { Force per } \\
\text { block (N) }\end{array}$ & $\begin{array}{l}\text { Force per } \\
\text { block (lbf) }\end{array}$ & $\begin{array}{l}\text { Force full } \\
\text { length (N) }\end{array}$ & $\begin{array}{l}\text { Force full } \\
\text { length (lbf) }\end{array}$ \\
\hline p1 & $(-3401,5074)$ & $(-207,309)$ & $(-46.6,69.6)$ & $(-415,619)$ & $(-93.3,139.2)$ \\
\hline p2 & $(-6006,-1204)$ & $(-366,-73)$ & $(-82.4,-16.5)$ & $(-733,-147)$ & $(-164.7,-33)$ \\
\hline p3 & $(-1204,-6006)$ & $(-73,-366)$ & $(-16.5,-82.4)$ & $(-147,-733)$ & $(-33,-164.7)$ \\
\hline p4 & $(5074,-3401)$ & $(309,-207)$ & $(69.6,-46.6)$ & $(619,-415)$ & $(139.2,-93.3)$ \\
\hline p5 & $(5074,3401)$ & $(309,207)$ & $(69.6,46.6)$ & $(619,415)$ & $(139.2,93.3)$ \\
\hline p6 & $(-1204,6006)$ & $(-73,366)$ & $(-16.5,82.4)$ & $(-147,733)$ & $(-33,164.7)$ \\
\hline p7 & $(-6006,1204)$ & $(-366,73)$ & $(-82.4,16.5)$ & $(-733,147)$ & $(-164.7,33)$ \\
\hline p8 & $(-3401,-5074)$ & $(-207,-309)$ & $(-46.6,-69.6)$ & $(-415,-619)$ & $(-93.3,-139.2)$ \\
\hline p9 & $(3401,-5074)$ & $(207,-309)$ & $(46.6,-69.6)$ & $(415,-619)$ & $(93.3,-139.2)$ \\
\hline p11 & $(6006,1204)$ & $(366,73)$ & $(82.4,16.5)$ & $(733,147)$ & $(164.7,33)$ \\
\hline p12 & $(1204,6006)$ & $(73,366)$ & $(16.5,82.4)$ & $(147,733)$ & $(33,164.7)$ \\
\hline p13 & $(-5074,3401)$ & $(-309,207)$ & $(-69.6,46.6)$ & $(-619,415)$ & $(-139.2,93.3)$ \\
\hline p14 & $(-5074,-3401)$ & $(-309,-207)$ & $(-69.6,-46.6)$ & $(-619,-415)$ & $(-139.2,-93.3)$ \\
\hline p15 & $(1204,-6006)$ & $(73,-366)$ & $(16.5,-82.4)$ & $(147,-733)$ & $(33,-164.7)$ \\
\hline p16 & $(6006,-1204)$ & $(366,-73)$ & $(82.4,-16.5)$ & $(733,-147)$ & $(164.7,-33)$ \\
\hline
\end{tabular}


The table below shows the total force vector on each half of the QD magnet when they are in contact.

\begin{tabular}{|l|l|l|l|l|l|}
\hline Subassembly & $\begin{array}{l}\text { Force per unit } \\
\text { length }(\mathrm{N} / \mathrm{m})\end{array}$ & $\begin{array}{l}\text { Force per } \\
\text { layer }(\mathrm{N})\end{array}$ & $\begin{array}{l}\text { Force per } \\
\text { layer (lbf) }\end{array}$ & $\begin{array}{l}\text { Force full } \\
\text { length (N) }\end{array}$ & $\begin{array}{l}\text { Force full } \\
\text { length (lbf) }\end{array}$ \\
\hline Left half & $(11075,0)$ & $(676,0)$ & $(151.9,0)$ & $(1351,0)$ & $(303.7,0)$ \\
\hline Right half & $(-11075,0)$ & $(-676,0)$ & $(-151.9,0)$ & $(-1351,0)$ & $(-303.7,0)$ \\
\hline
\end{tabular}

